\title{
The application of autologous platelet-rich plasma gel in cartilage regeneration
}

\author{
AIGUO XIE ${ }^{1}$, LANJUN NIE $^{1}$, GAN SHEN $^{1}$, ZIWEI CUI $^{2}$, PENG XU $^{2}$, HUAQIANG GE $^{2}$ and QIAN TAN ${ }^{2}$ \\ ${ }^{1}$ Department of Plastic Surgery, Second Affiliated Hospital of Nanjing Medical University; \\ ${ }^{2}$ Department of Plastic Surgery, Drum Tower Clinical Medical College, \\ Nanjing Medical University, Nanjing, Jiangsu 210008, P.R. China
}

Received November 5, 2013; Accepted June 16, 2014

DOI: $10.3892 / \mathrm{mmr} .2014 .2358$

\begin{abstract}
Cartilage defect caused by disease or trauma remains a challenge for surgeons, owning to the limited healing capacity of cartilage tissues. Cartilage tissue engineering provides a novel approach to address this issue, and appears promising for patients with cartilage defects. The cell scaffold, as one of the three key elements of tissue engineering, plays an important role in cartilage tissue engineering. Platelet-rich plasma (PRP), which is a fraction of the plasma containing multiple growth factors, has become a major research focus in the context of its use as a bioactive scaffold for tissue engineering. Therefore, we investigated the value of using PRP scaffolds combined with chondrocytes in cartilage tissue engineering. In this study, we examined the levels of growth factors in PRP, and the effects of PRP on cell proliferation and matrix synthesis in rabbit chondrocytes cultured in PRP. Short-term in vitro culture followed by long-term in vivo implantation was performed to evaluate the chondrogenesis of neocartilage in vivo. The results show that PRP may provide a suitable environment for the proliferation and maturation of chondrocytes, and can be used as a promising bioactive scaffold for cartilage regeneration.
\end{abstract}

\section{Introduction}

The management of various cartilage lesions remains a challenge for surgeons, due to the limited regenerative capacity of cartilage tissues. A number of echniques have been developed for the treatment of cartilage defects during the past decade, aiming to address this complex issue. These techniques, including autologous cartilage implantation and autologous

Correspondence to: Dr Qian Tan, Department of Plastic Surgery, Drum Tower Clinical Medical College, Nanjing Medical University, 321 Zhong Shan Road, Nanjing, Jiangsu 210008, P.R. China

E-mail:smmutanqian@sina.com

Key words: platelet-rich plasma, scaffold, growth factors, cartilage engineering chondrocyte implantation, have been used to repair cartilage defects. More recently, tissue engineering has been reported as a promising approach to repair cartilage defects. This technique involves harvesting a high number of cells from the human body and seeding them on a biodegradable scaffold, and then implanting these scaffolds to cover the defective areas. Therefore, the emergence of cartilage tissue engineering techniques provides potential solutions for the clinical repair of cartilage defects. In addition, seeding of chondrocytes on biodegradable scaffolds in order to allow formation of a three-dimensional cartilage tissue for implantation is a primary approach in cartilage tissue engineering aiming to repair cartilage defects. Cao et al (1) used a chondrocyte-polymer composite to produce tissue-engineered cartilage in the shape of a human ear. Luo et al (2) reconstructed a segmental tracheal defect by pedicled tissue-engineered trachea in rabbits.

A variety of biodegradable materials has been explored for eventual use as scaffolds in cartilage tissue engineering, including collagen gels and sponges, hyaluronic acid matrices, and poly( $\alpha$-hydroxyesters), such as polyglycolic acid (PGA), polylactic acid (PLA) and their copolymers. $\mathrm{Wu}$ et al (3) were able to form mature cartilage tissue by using poly(hydroxybutyrate-co-hydroxyvalerate) (PHBV), and improved the PHBV scaffold with bioglass to produce cartilage tissue with improved biomechanical and biochemical properties. Xue et al (4) reported that chondrogenesis of bone marrow-derived stromal cells seeded on PGA promotes regeneration of cartilage. However, the natural and artificial synthetic material used in these studies are still exogenous, often causing inflammatory and immune reactions. Therefore, research on biodegradable scaffolds produced by autologous material is receiving increasing attention. Platelet-rich plasma (PRP) is ideal for use as a biomedical material for tissue engineering due to its reduced immunogenicity and improved biocompatibility, in addition to its high content in growth factors.

Numerous studies have used PRP as a scaffold for bone tissue engineering. El Backly et al (5) used platelet-rich plasma to enhance the osteoconductive properties of a hydroxyapatite- $\beta$-tricalcium phosphate scaffold for late healing of critical-size rabbit calvarial defects. Jiang et al (6) repaired calvarial defects in rabbits using platelet-rich plasma as the scaffold, which carried bone marrow stromal cells. 
However, up to now, few studies have investigated the utility of autologous platelet-rich plasma gel (APG) in cartilage tissue engineering. Therefore, in this study, APG was prepared and studied. The chondrocytes were seeded on the scaffolds and cultured in vitro for one week, followed by in vivo implantation for six weeks, to observe the growth and proliferation of the chondrocytes and the engineered cartilage tissue in PRP. The level of growth factors, cell proliferation in the scaffold, extracellular matrix production, as well as biomechanical properties of the neocartilage were examined, to evaluate the value of the PRP scaffold in cartilage regeneration.

\section{Materials and methods}

Ethics statement. All experimental procedures conducted on animals in this study were approved by the Ethics Committee of the Nanjing Medical University.

Blood collection and cell isolation. New Zealand white rabbits (2-3 months old), obtained from the Animal Center of Nanjing Medical University (Nanjing, China) were used to isolate PRP and articular cartilage. Animals were first anesthetized by intramuscular injection of sodium pentobarbital $(30 \mathrm{mg} / \mathrm{kg})$ and ketamine hydrochloride $(30 \mathrm{mg} / \mathrm{kg})$. Blood $(50 \mathrm{ml})$ was collected from the posterior auricular vein with a $14-\mathrm{G}$ needle using a 50-ml syringe treated with an anti-coagulant citrate dextrose solution (Sigma-Aldrich, St. Louis, MO, USA). Following collection of peripheral blood, the animals were sacrificed by intravenously injecting supersaturated pentobarbital.

The articular cartilages were removed from the knees and hip joints of the rabbits and were cut into small pieces as described in (3). Chondrocytes were released from the cartilage slices by digesting with collagenase II $(0.2 \% \mathrm{w} / \mathrm{v}$; Invitrogen Life Technologies, Carlsbad, CA, USA). The isolated cells were then cultured in Dulbecco's modified Eagle's medium (DMEM) supplemented with $10 \%$ fetal calf serum (FCS; Gibco Life Technologies, Carlsbad, CA, USA), 100 U/ml penicillin and $100 \mu \mathrm{g} / \mathrm{ml}$ streptomycin (Sigma-Aldrich, Irvine, UK). Cells were incubated at $37^{\circ} \mathrm{C}$ in a $5 \% \mathrm{CO}_{2}$ incubator, and the medium was changed every 3 days.

Preparation of PRP. PRP was isolated from fresh rabbit blood $(\sim 50 \mathrm{ml})$ as previously described (7). Briefly, the blood was treated with anti-coagulant and was separated into the plasma and the hemocyte (erythrocytes and leukocytes) fractions, and then separated into PRP by continuous two-step sedimentation (Fig. 1A). PRP was clotted by adding a 10\% (v/v) thrombin solution in $\mathrm{CaCl}_{2}\left(1,000 \mathrm{U} / \mathrm{ml}\right.$ thrombin in $\left.100 \mathrm{mM} \mathrm{CaCl}_{2}\right)$ to yield a final thrombin concentration of $100 \mathrm{U} / \mathrm{ml}$. Soluble PRP was released from the clotted preparations by centrifugation at $1,500 \mathrm{~g}$ for $5 \mathrm{~min}$.

Quantification of growth factors. The level of transforming growth factor- $\beta$ (TGF- $\beta$ ), insulin-like growth factor (IGF), epidermal growth factor (EGF) and platelet-derived growth factor (PDGF)-AB in whole blood, PRP and activated PRP (aPRP) was determined using corresponding enzyme-linked immunosorbent assay (ELISA) kits according to the manufacturer's instructions (R\&D Systems, Inc., Minneapolis, MN) as in (8).
Cell cultures with different concentrations of PRP and cell proliferation assay. Cell cultures in DMEM fetal calf serum-free medium (SFM) containing different concentrations of PRP $(0,5,10,20$ and $30 \%)$ were used to test the effect of PRP on the proliferation of chondrocytes. Briefly, the chondrocytes at passage 2 were precultured in DMEM SFM for $24 \mathrm{~h}$. The chondrocytes were then cultured under five different conditions: 0\% PRP (SFM without PRP), 5\% PRP (5\% PRP in SFM), $10 \%$ PRP (10\% PRP in SFM), 20\% PRP (20\% PRP in SFM), and 30\% PRP (30\% PRP in SFM). The proliferation of the chondrocytes was assessed with a cell counting kit (CCK)-based colorimetric assay as in (9), following the manufacturer's instructions (CCK-8 kit; Rockville, MD, USA). The optical density (OD) of the samples was measured on a DU800 spectrophotometer (Beckman Coulter, Brea, CA, USA).

Formation of chondrocyte/PRP composites. After subculturing twice, the chondrocyte suspension at passage 2 was collected by trypsin digestion and centrifuged at $352 \mathrm{x}$ g for $5 \mathrm{~min}$. The chondrocytes were washed 2 times with phosphate-buffered saline (PBS) to remove the residual serum, and cells were counted and resuspended in PRP, at a density of $5.0 \times 10^{7}$ cells $/ \mathrm{ml}$. To activate PRP, $0.15 \mathrm{ml}$ of a $\mathrm{CaCl}_{2}$ and $\mathrm{Gibco}^{\circledR}$ bovine thrombin mixture $\left(1,000 \mathrm{U} / \mathrm{ml}\right.$ in $100 \mathrm{mg} / \mathrm{ml} \mathrm{CaCl}_{2}$; Thermo Fisher Scientific, Waltham, MA, USA) was added to the cell/PRP suspension. Seven days later, the composites were subcutaneously implanted into BALB-c-nu nude mice (4 weeks old, $20 \mathrm{~g}$ ), obtained from the Animal Center of Nanjing Medical University. The composites were harvested at 6 weeks post-implantation.

Scanning electron microscopy (SEM) of in vitro-engineered tissue. The attachment, proliferation and matrix production of the cells on the scaffolds were examined using light microscopy (Eclipse TS100 microscope; Nikon, Tokyo, Japan) and SEM (XL-30; Philips, Amsterdam, The Netherlands).

\section{Evaluation of in vivo-engineered tissue}

Gross observation of in vivo-engineered tissue. The composites were harvested at 6 weeks after implantation and were recorded by image analysis.

Histological evaluation. Following implantation for 6 weeks, representative in vivo-formed cartilaginous tissue was fixed in neutral-buffered formalin, embedded in paraffin and sectioned (5- $\mu \mathrm{m}$ thick sections). The cross-sections were stained with hematoxylin and eosin $(\mathrm{H} \& \mathrm{E})$ and safranine $\mathrm{O}$ (Sigma-Aldrich, St. Louis, MO, USA).

Five-micrometer cryosections were used for immunostaining with the collagen type II monoclonal mouse anti-rabbit antibody (Santa Cruz Biotechnology, Inc., Santa Cruz, CA, USA). The samples were immersed in PBS containing $1 \%$ goat serum at $4^{\circ} \mathrm{C}$ overnight, in order to block the non-specific reactions. Subsequently, the sections were incubated in PBS containing 1\% bovine serum albumin (BSA) and anti-collagen type II antibody (1:100 working dilution) at $25^{\circ} \mathrm{C}$ for $4 \mathrm{~h}$. After washing with PBS three times, the samples were incubated in PBS containing $3 \%$ BSA. Finally, the samples were incubated in PBS containing $1 \%$ BSA and horseradish peroxidase (HRP)-conjugated anti-rabbit $\mathrm{IgG}$ antibody (1:150 working dilution; Santa Cruz Biotechnology, Inc.) at $25^{\circ} \mathrm{C}$ for $4 \mathrm{~h}$, followed by color 


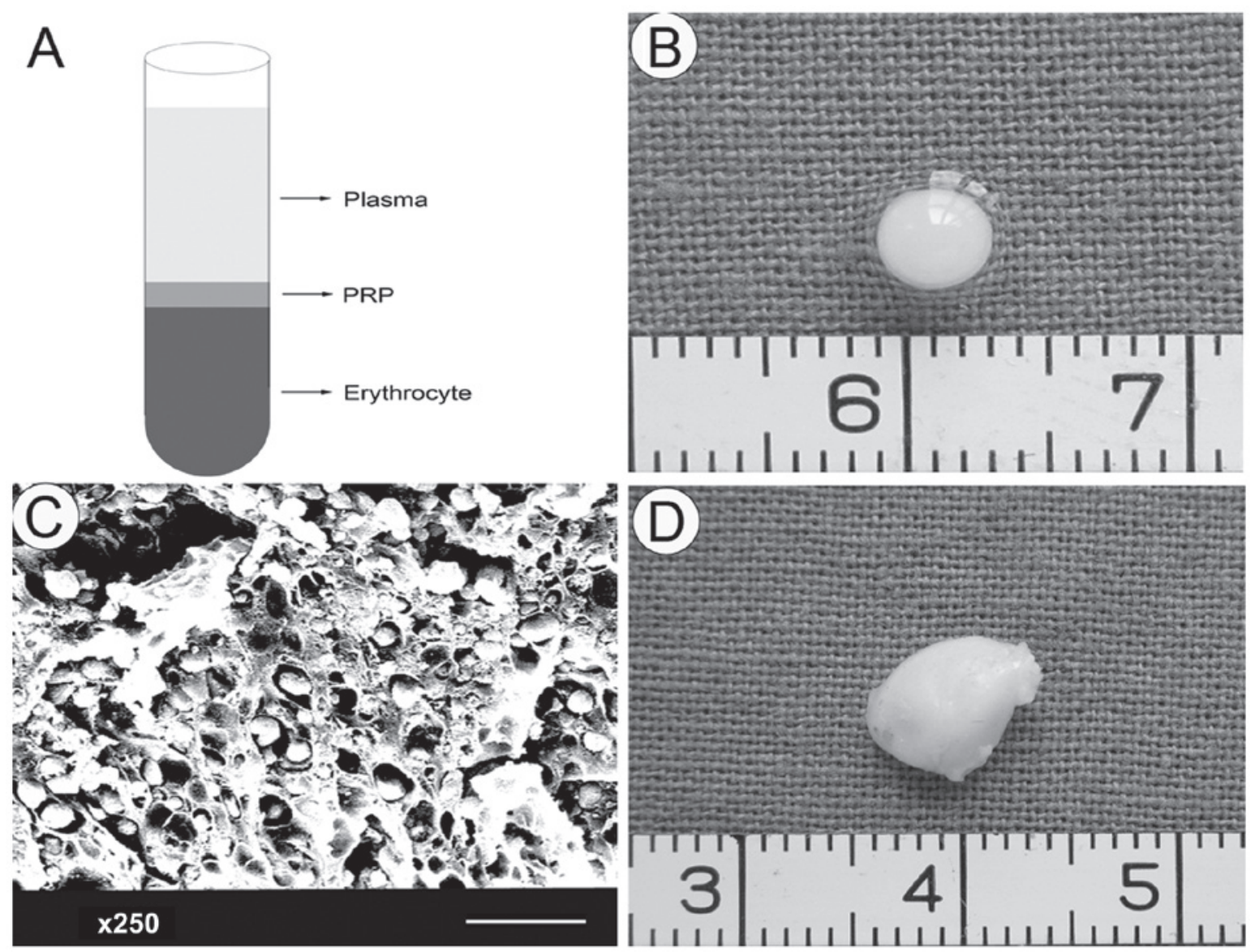

Figure 1. The optical and scanning electron microscopy (SEM) micrographs of the chondrocyte-autologous platelet-rich plasma gel (APG) composite. (A) Different fractions of the blood during platelet-rich plasma (PRP) preparation. (B) In the composite, the tissue is ivory-white and cartilage-like after 7-day culture in vitro. (C) SEM shows that scaffolds have a macroporous structure with open pores, and that chondrocytes grow well in APG after 3 days of in vitro culture. Scale bar, $100 \mu \mathrm{m}$. (D) Gross view of the composite after 6 weeks culture in vivo.

development with diaminobenzidine tetrahydrochloride (DAB; Santa Cruz Biotechnology, Inc), as in (3).

Quantitative analysis of in vivo cartilage formation. After 6 weeks of in vivo culture, the glycosaminoglycan (GAG) content $(10,11)$ and the total collagen content were analyzed according to previously described methods (12). Briefly, the protein solutions of the specimens were prepared. The specific binding of Alcian Blue and polysulfated molecules of GAGs in cartilage were acheived by adding a series of reagents. All GAGs were precipitated specifically in guanidine- $\mathrm{HCl}$ by using a low $\mathrm{pH}$ in combination with detergent and high salt concentration. The precipitate was dissolved in a mixture of guanidine- $\mathrm{HCl}$ and propanol. The optical density (OD) of the samples was measured, and a linear standard curve between 0.5 and $20 \mathrm{mg}$ was generated by adding known amounts of proteoglycans.

Biomechanical analysis. A biomechanical analyzer (Instron, Norwood, MA, USA) was used for the biomechanical tests. As previously described (3), a constant compressive strain rate of $1 \mathrm{~mm} / \mathrm{min}$ was applied, until a maximal force of $100 \mathrm{~N}$ was achieved, and thus a force-displacement curve was obtained. The compressive modulus of the tested tissue was calculated based on the force-displacement curve.

Statistical analysis. All data were expressed as means \pm standard deviation (SD), with $\mathrm{n}=6$. The differences were analyzed with a one-way analysis of variance (ANOVA), using SPSS for Windows software, version 13.0 (SPSS, Inc., Chicago, IL, USA). A P-value $<0.05$ was considered to indicate a statistically significant difference.

\section{Results}

Cell cultures and chondrocyte-APGcompositeformation.After 3 days of primary culture, cells reached $85-90 \%$ confluence. Following two additional subculturing rounds, the cells maintained their morphology, and chondrocytes at passage 2 were used for cartilage engineering. The chondrocyte-APG composite had an ivory-whitish appearance (Fig. 1B and D). When observed by SEM, the chondrocytes grew well in the APG scaffold, and the scaffold exhibited a macroporous structure with interconnected open pores, of size varying from 30 to $300 \mu \mathrm{m}$ (Fig. 1C).

Quantification of growth factors. The results of ELISA assays showed that the level of TGF- $\beta 1$ increased from $3.10 \pm 0.43 \mathrm{ng} / \mathrm{ml}$ in whole blood to $5.9 \pm 0.67 \mathrm{ng} / \mathrm{ml}$ in PRP and $13.55 \pm 1.53 \mathrm{ng} / \mathrm{ml}$ in aPRP (Fig. 2A). The concentration of IGF showed an increase from $14.50 \pm 1.63 \mathrm{ng} / \mathrm{ml}$ in whole-blood to $45.03 \pm 4.67 \mathrm{ng} / \mathrm{ml}$ in PRP and $110.06 \pm 12.53 \mathrm{ng} / \mathrm{ml}$ in aPRP (Fig. 2B). The concentration of PDGF-AB showed an increase from $145.02 \pm 16.3 \mathrm{pg} / \mathrm{ml}$ in whole blood to $263.00 \pm 26.71 \mathrm{pg} / \mathrm{ml}$ in PRP and $503.00 \pm 53.00 \mathrm{pg} / \mathrm{ml}$ in 

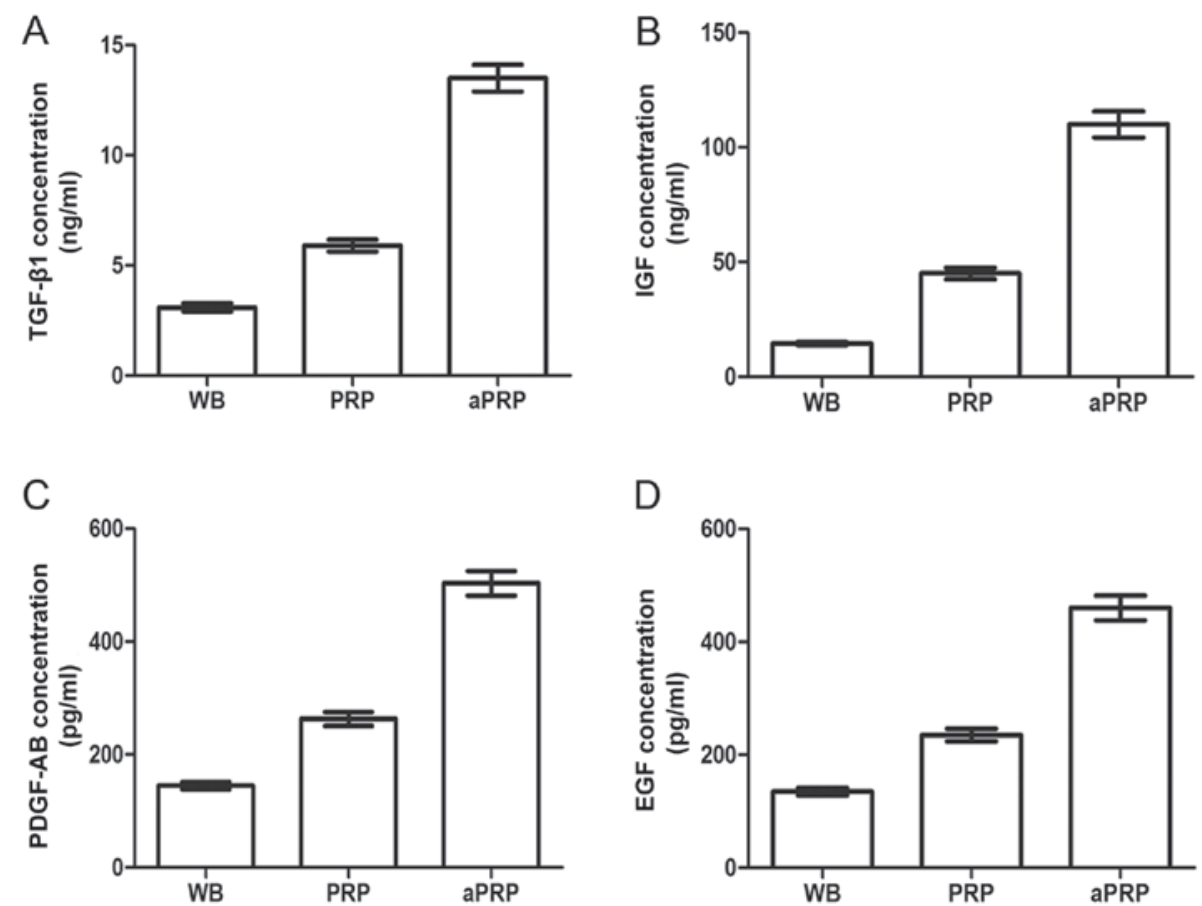

Figure 2. Quantification of growth factors. The concentration of transforming growth factor- $\beta$ (TGF- $\beta$ ), insulin-like growth factor (IGF), epidermal growth factor (EGF) and platelet-derived growth factor (PDGF)-AB is increased in whole blood (WB), platelet-rich plasma (PRP), and activated PRP (aPRP).
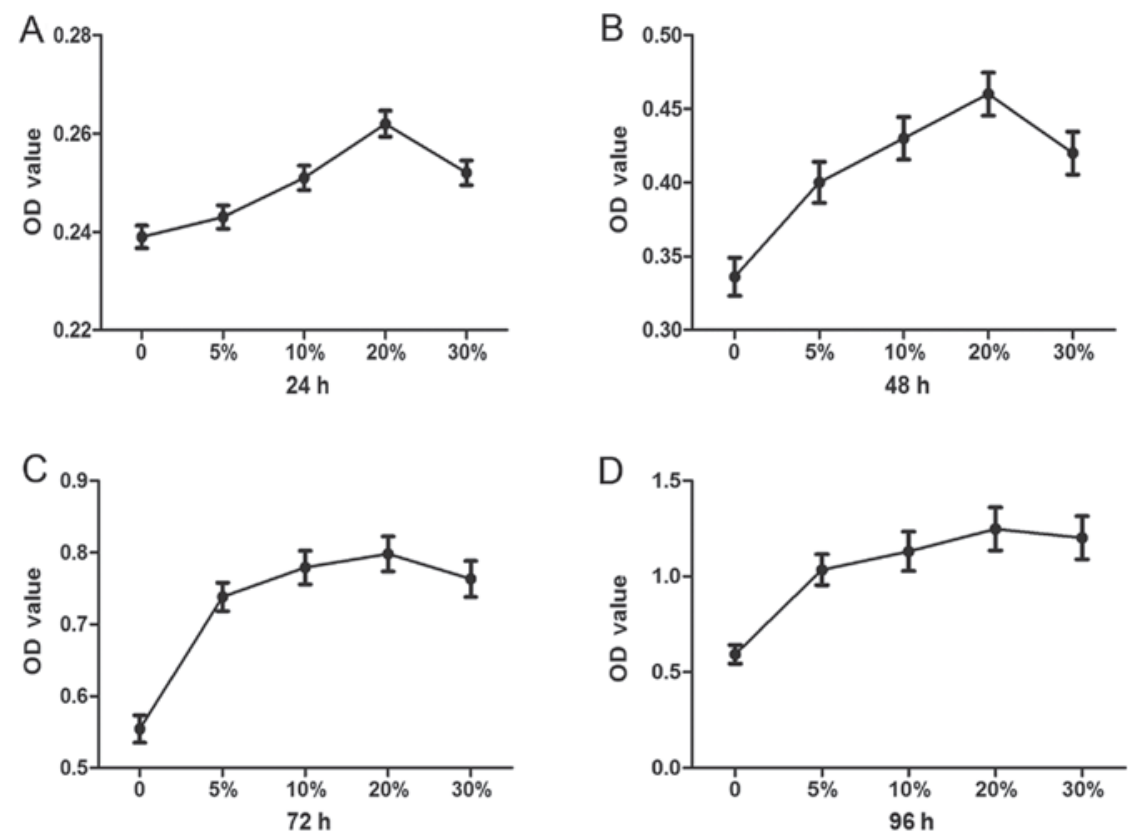

Figure 3. Cell proliferation in the presence of different concentrations of platelet-rich plasma (PRP). Cell proliferation increases at different concentrations of PRP, and reaches a peak at $20 \%$ PRP. OD, optical density.

aPRP (Fig. 2C). The concentration of EGF showed an increase from $135.12 \pm 16.3 \mathrm{pg} / \mathrm{ml}$ in whole blood to $235.30 \pm 25.67 \mathrm{pg} / \mathrm{ml}$ in PRP and $460.23 \pm 50.98 \mathrm{pg} / \mathrm{ml}$ in aPRP (Fig. 2D).

The effect of different concentrations of PRP on cell proliferation. The result of the CCK assay showed that different concentrations of PRP have different effects on cell proliferation. Cell proliferation in the presence of different concentrations of PRP $(0,5,10,20$ and $30 \%)$ gradually increased, and reached a peak at 20\% PRP (Fig. 3).

\section{Evaluation of in vivo-engineered tissue}

Gross evaluation of the in vivo-engineered composites. The chondrocyte-APG composites maintained their original size and presented a cartilage-like appearance following in vivo implantation for 6 weeks. 


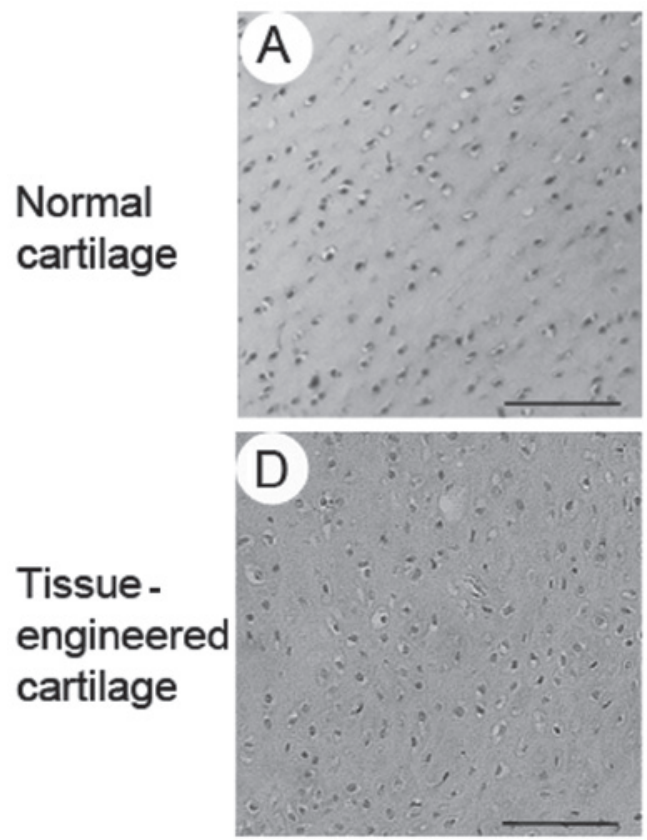

$\mathrm{H} \& \mathrm{E}$
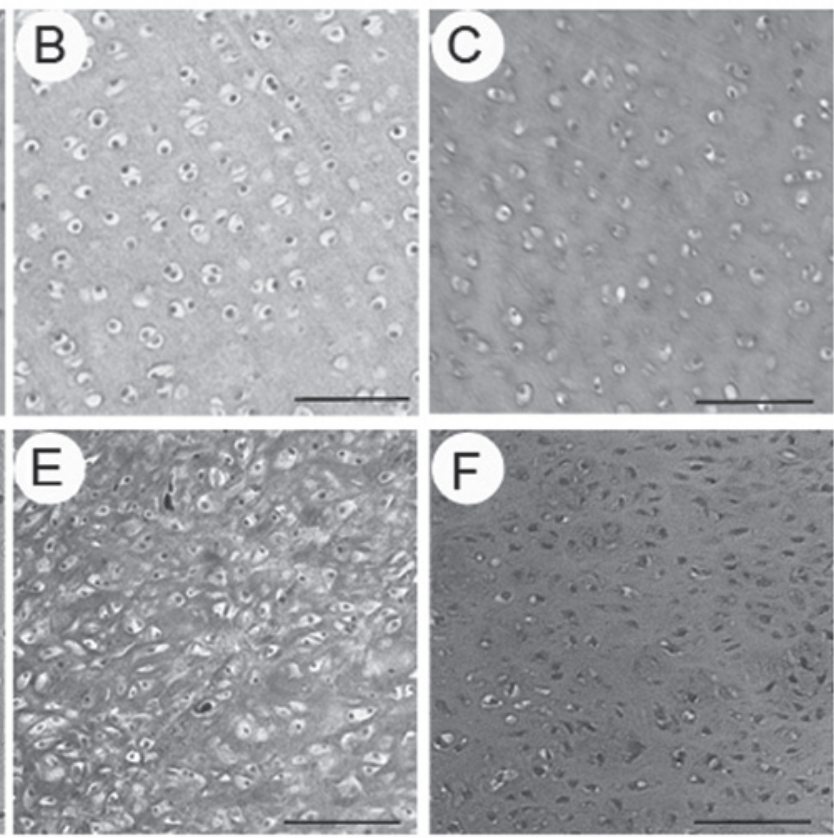

Collagen-II

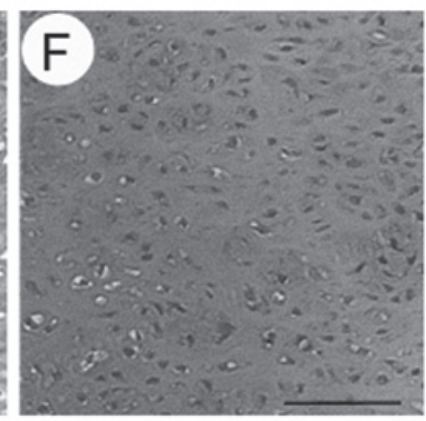

Safranine 0

Figure 4. Histological and immunohistochemical examination of in vivo-engineered cartilage. (A-C) Normal cartilage and (D-F) tissue-engineered cartilage, (A and D) stained with H\&E, (B and E) immunostained with collagen type II antibody, and (C and F) stained with safranine O. The chondrocyte-APG composite forms cartilage-like tissue. Lacuna-like structures, positive safranine O staining and type II collagen immunostaining are observed in the tissue-engineered cartilage, similar to the normal cartilage. Scale bar, $100 \mu \mathrm{m}$. H\&E, hematoxylin and eosin; APG, autologous platelet-rich plasma gel.
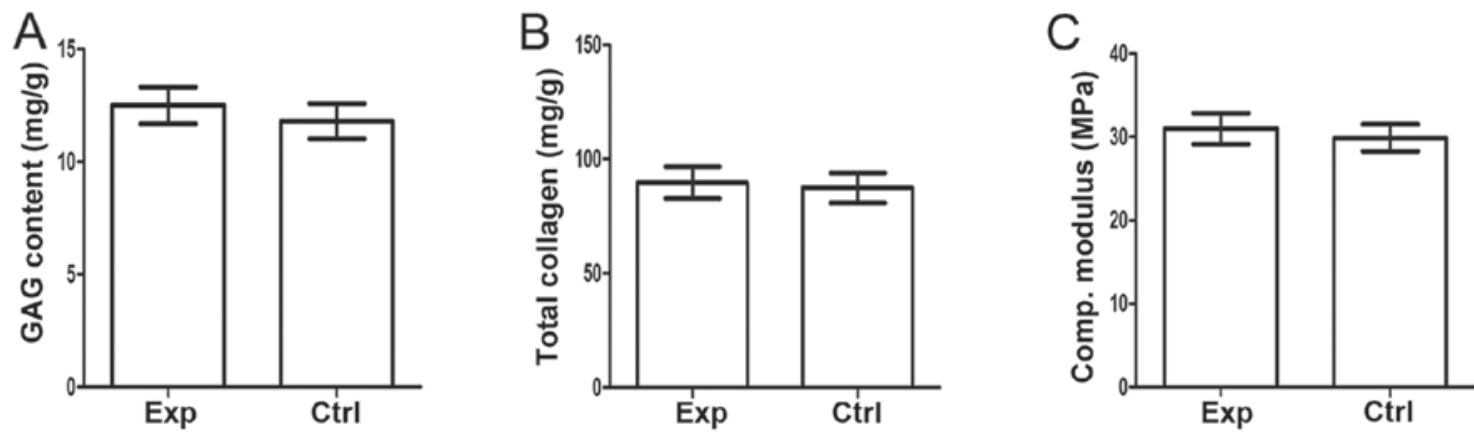

Figure 5. (A) Collagen content, (B) glycosaminoglycan (GAG) content and (C) compressive modulus in the tissue-engineered experimental group (Exp) and the normal cartilage control group (Ctrl). There is no significant difference in the collagen and GAG content, as well as in the compressive modulus between tissue-engineered and normal cartilage $(\mathrm{P}>0.05)$.

Histology and immunohistochemistry. Histology and immunohistochemistry revealed the formation of tissue-engineered cartilage. Formation of the cartilage-like tissue was observed, with an obvious lacuna structure and positive staining for safranine $\mathrm{O}$ and type II collagen (Fig. 4).

Collagen and GAG content, and compressive modulus. Quantitative analysis of the collagen and GAG content demonstrated that there is no significant difference $(\mathrm{P}>0.05)$ on extracellular matrix (ECM) contents between normal and tissue-engineered cartilage (Fig. 5A and B).

The mechanical properties of the cartilage are of importance, and the collagen and GAG content only partly reflect these. Analysis of the compressive modulus further indicated that there is no significant difference $(\mathrm{P}>0.05)$ between tissue-engineered and normal cartilage (Fig. 5C).

\section{Discussion}

Cartilage defects have been a challenge for surgeons. However, developments in cartilage tissue engineering in the past few years have provided a means to repair cartilage defects (13-15). Certain cases of tissue-engineered cartilage implantation have been reported in the clinic (e.g., 16). The scaffold, which acts as a cell carrier, plays a significant role in the regeneration of the cartilage. Numerous natural or synthetic materials have been exploited as scaffolds during the past decade for cartilage tissue engineering. However, an optimal biodegradable scaffold for cartilage tissue engineering would ideally be less immunogenic than the currently used ones, with improved biocompatibility and an adequate degradation rate; in addition, the scaffold 
is expected to promote attachment, proliferation and the activity of cells (17).

Autologous platelet-rich plasma gel (APG) is a fraction of the plasma containing multiple growth factors, such as TGF- $\beta 1$, PDGF, EGF and IGF-1, and which can induce biological changes in cell proliferation and matrix metabolism in a variety of connective tissues $(18,19)$. APG can be produced by centrifugal separation of autologous whole-blood samples (20). Moreover, APG has an ideal three-dimensional structure, reduced immunogenicity and satisfactory biocompatibility. Scanning electron microscope analysis in this study revealed that APG has a three-dimensional nanostructure, and that cells grew well in APG. Due to the above advantages, APG appears promising as a novel biodegradable scaffold to be used in tissue engineering.

Despite the development of various natural or synthetic biomaterials for cartilage tissue engineering, the biological safety of scaffolds and the long-term biocompatibility between chondrocytes and scaffolds, or between tissue-engineered cartilage and acceptor tissue, still needs to be further investigated. Most of the currently used biodegradable material has been reported to cause certain inflammatory reactions, and in some cases, it is even rejected following implantation via immune reactions $(21,22)$. Compared to other materials, APG is an autologous material with reduced immunogenicity and enhanced biocompatibility, and has been used in certain clinical cases. Torrero et al (23) treated 30 patients (18-65 years old, diagnosed with Outerbridge grade I-III chondropathy in the knee, pain for more than 3 months following conservative treatment, and no bone axial defects) affected by chondropathy of the knee with a single intrarticular injection of PRP, and statistical analysis with ANOVA showed significantly better $(\mathrm{P}<0.05)$ results in these patients in terms of KOOS and VAS scores at 1, 3 and 6 months, in comparison to the pre-injection values. Redaelli et al (24) revitalized face and neck with PRP injection and also obtained satisfactory results.

In addition, PRP provides a high level of growth factors such as TGF- $\beta 1$, FGF etc. In the present study, we measured the concentration of growth factors in the whole blood, PRP and aPRP. The results showed higher concentrations of growth factors in PRP than that in the whole blood. Moreover, the activated PRP in APG showed the highest concentrations of growth factors among the three groups. The reason for that is that the platelets collected in PRP are activated by the addition of thrombin and calcium chloride, which induces the release of these factors from the $\alpha$ granules $(25,26)$.

It was reported that most growth factors found in PRP can enhance cell proliferation and/or matrix production (18). TGF- $\beta$ plays an important role in tissue regeneration, cell differentiation and embryonic development, and the application of TGF- $\beta$ on the chondrocytes induces the synthesis of type I relative to type II collagen $(27,28)$. Both IGF-I and IGF-II stimulate chondrocyte proliferation, and maintain the chondrocyte phenotype (29). We showed in this study that PRP, a highly concentrated natural combination of growth factors, has the ability to stimulate cell proliferation. Furthermore, we studied the effect of different concentrations of PRP on cell proliferation, and the result showed that cell proliferation is dose- and time-dependently increased by PRP, with the highest levels of cell proliferation observed at a dose of $20 \%$
PRP. In addition to proliferation, the growth factors present in PRP are known to accelerate epithelial regeneration, promote angiogenesis, and ultimately improve wound healing in skin and periodontal tissue (30).

In conclusion, PRP provides a good environment for cell growth and proliferation, by supplementing the chondrocytes with all the essential nutrients. In this study, the chondrocyte-APG composite was formed on an ivorywhitish, cartilage-like tissue in vitro and in vivo. Histological and immunohistochemical examination of the engineered cartilage showed that the majority of the cells formed lacunalike structures, with positive staining for safranine $\mathrm{O}$ and type II collagen.

In addition, there was no significant difference observed in the compressive modulus between the tissue-engineered and the normal cartilage groups. The mechanical properties of neocartilage tissue are determined by the content of the ECM (31). Previous studies reported that the ECM content (GAG and total collagen) in cartilage tissue contributes to the improvement of mechanical strength (e.g., 32). Our study also found that the GAG and total collagen content in tissue-engineered cartilage is similar to that in the normal cartilage tissue. Thus, the APG scaffold supported the growth of cells, which maintained their activity, fully expressed their phenotype, produced the extracellular matrix, and preserved the characteristic spherical morphology associated with the synthesis of type II collagen and cartilage-specific proteoglycans.

In summary, the APG scaffolds provide a high level of growth factors, which can efficiently promote chondrocyte proliferation. The chondrocyte-scaffold composites formed cartilage-like tissue of homogeneous structure, showing strong mechanical properties and high cartilage matrix content. Overall, these results suggest that the APG scaffolds may constitute a suitable biodegradable scaffolds for cartilage tissue engineering.

\section{Acknowledgements}

This study was supported by a research grant from the National Natural Science Foundation of China (no. 81272108).

\section{References}

1. Cao Y, Vacanti JP, Paige KT, Upton J and Vacanti CA: Transplantation of chondrocytes utilizing a polymer-cell construct to produce tissue-engineered cartilage in the shape of a human ear. Plast Reconstr Surg 100: 294-303, 1997.

2. Luo X, Liu Y,Zhang Z, et al: Long-term functional reconstruction of segmental tracheal defect by pedicled tissue-engineered trachea in rabbits. Biomaterials 34: 3336-3344, 2013.

3. Wu J, Xue K, Li H, Sun J and Liu K: Improvement of PHBV scaffolds with bioglass for cartilage tissue engineering. PloS One 8: e71563, 2013.

4. Xue K, Zhu Y, Zhang Y, Chiang C, Zhou G and Liu K: Xenogeneic chondrocytes promote stable subcutaneous chondrogenesis of bone marrow-derived stromal cells. Int J Mol Med 29: 146-152, 2012.

5. El Backly RM, Zaky SH, Canciani B, et al: Platelet rich plasma enhances osteoconductive properties of a hydroxyapatite- $\beta$-tricalcium phosphate scaffold $\left(\right.$ Skelite $\left.^{\mathrm{TM}}\right)$ for late healing of critical size rabbit calvarial defects. J Craniomaxillofac Surg: Aug 7, 2013 (Epub ahead of print).

6. Jiang ZQ, Liu HY, Zhang LP, Wu ZQ and Shang DZ: Repair of calvarial defects in rabbits with platelet-rich plasma as the scaffold for carrying bone marrow stromal cells. Oral Surg Oral Med Oral Pathol Oral Radiol 113: 327-333, 2012. 
7. Kanthan SR, Kavitha G, Addi S, Choon DS and Kamarul T: Platelet-rich plasma (PRP) enhances bone healing in non-united critical-sized defects: a preliminary study involving rabbit models. Injury 42: 782-789, 2011.

8. Kilroy GE, Foster SJ, Wu X, et al: Cytokine profile of human adipose-derived stem cells: expression of angiogenic, hematopoietic, and pro-inflammatory factors. J Cell Physiol 212: 702-709, 2007

9. Xue K, Qi L,Zhou G and Liu K: Atwo-step method of constructing mature cartilage using bone marrow-derived mesenchymal stem cells. Cells Tissues Organs 197: 484-495, 2013

10. Björnsson S: Simultaneous preparation and quantitation of proteoglycans by precipitation with alcian blue. Anal Biochem 210: 282-291, 1993.

11. Zhou G1, Liu W, Cui L, Wang X, Liu T and Cao Y: Repair of porcine articular osteochondral defects in non-weightbearing areas with autologous bone marrow stromal cells. Tissue Eng 12 : 3209-3221, 2006.

12. Reddy GK and Enwemeka CS: A simplified method for the analysis of hydroxyproline in biological tissues. Clin Biochem 29: 225-229, 1996.

13. Kuhne M, John T, El-Sayed K, et al: Characterization of auricular chondrocytes and auricular/articular chondrocyte co-cultures in terms of an application in articular cartilage repair. Int $\mathrm{J}$ Mol Med 25: 701-708, 2010.

14. Moon MH, Jeong JK, Lee YJ, Seol JW and Park SY: Sphingosine-1-phosphate inhibits interleukin-1 $\beta$-induced inflammation in human articular chondrocytes. Int J Mol Med 30 $1451-1458,2012$.

15. Schubert T, Anders S, Neumann E, et al: Long-term effects of chondrospheres on cartilage lesions in an autologous chondrocyte implantation model as investigated in the SCID mouse model. Int J Mol Med 23: 455-460, 2009.

16. Sharma B, Fermanian S, Gibson M, et al: Human cartilage repair with a photoreactive adhesive-hydrogel composite. Sci Trans Med 5: 167ra166, 2013

17. Vinatier C, Bouffi C, Merceron C, et al: Cartilage tissue engineering: towards a biomaterial-assisted mesenchymal stem cell therapy. Curr Stem Cell Res Ther 4: 318-329, 2009.

18. Akeda K, An HS, Okuma M, et al: Platelet-rich plasma stimulates porcine articular chondrocyte proliferation and matrix biosynthesis. Osteoarthritis Cartilage 14: 1272-1280, 2006.

19. Staudenmaier R, Froelich K, Birner M, et al: Optimization of platelet isolation and extraction of autogenous TGF-beta in cartilage tissue engineering. Artif Cells Blood Substit Immobil Biotechnol 37: 265-272, 2009.

20. Wu W, Zhang J, Dong Q, Liu Y, Mao T and Chen F: Platelet-rich plasma - a promising cell carrier for micro-invasive articular cartilage repair. Med Hypotheses 72: 455-457, 2009.
21. Arora R, Milz S, Sprecher C, Sitte I, Blauth M and Lutz M: Behaviour of ChronOS Inject in metaphyseal bone defects of distal radius fractures: tissue reaction after 6-15 months. Injury 43: 1683-1688, 2012.

22. Pascual G, Rodriguez M, Sotomayor S, Perez-Kohler B and Bellon JM: Inflammatory reaction and neotissue maturation in the early host tissue incorporation of polypropylene prostheses. Hernia 16: 697-707, 2012

23. Torrero JI, Aroles F and Ferrer D: Treatment of knee chondropathy with platelet rich plasma. Preliminary results at 6 months of follow-up with only one injection. J Biol Regul Homeost Agents 26 (2 Suppl 1): S71-S78, 2012.

24. Redaelli A, Romano D and Marciano A: Face and neck revitalization with platelet-rich plasma (PRP): clinical outcome in a series of 23 consecutively treated patients. J Drugs Dermatol 9: 466-472, 2010

25. Borrione P, Gianfrancesco AD, Pereira MT and Pigozzi F Platelet-rich plasma in muscle healing. Am J Phys Med Rehabil 89: 854-861, 2010.

26. Yu W, Wang J and Yin J: Platelet-rich plasma: a promising product for treatment of peripheral nerve regeneration after nerve injury. Int J Neurosci 121: 176-180, 2011.

27. Centrella M, McCarthy TL and Canalis E: Effects of transforming growth factors on bone cells. Connect Tissue Res 20 267-275, 1989.

28. Centrella M, McCarthy TL and Canalis E: Platelet-derived growth factor enhances deoxyribonucleic acid and collagen synthesis in osteoblast-enriched cultures from fetal rat parietal bone. Endocrinology 125: 13-19, 1989.

29. McCarthy TL, Centrella M and Canalis E: Regulatory effects of insulin-like growth factors I and II on bone collagen synthesis in rat calvarial cultures. Endocrinology 124: 301-309, 1989.

30. Canalis E, McCarthy TL and Centrella M: Effects of platelet-derived growth factor on bone formation in vitro. J Cell Physiol 140: 530-537, 1989.

31. Bastiaansen-Jenniskens YM, Koevoet W, de Bart AC, et al: Contribution of collagen network features to functional properties of engineered cartilage. Osteoarthritis Cartilage 16: 359-366, 2008.

32. Mikic B, Isenstein AL and Chhabra A: Mechanical modulation of cartilage structure and function during embryogenesis in the chick. Ann Biomed Eng 32: 18-25, 2004. 